\title{
Validação de um Sistema de Visualização de Algoritmos no Ensino de Provas por Indução em Teoria dos Grafos
}

\author{
Mateus O. Santos ${ }^{1}$, Joslaine C. J. Freitas ${ }^{1}$, \\ Gecirlei F. Silva ${ }^{1}$, Esdras L. Bispo Jr. ${ }^{1,2}$ \\ ${ }^{1}$ Instituto de Ciências Exatas e Tecnológicas \\ Universidade Federal de Jataí (UFJ) - Jataí, Goiás, Brasil \\ ${ }^{2}$ Jataí ACM SIGCSE Chapter \\ Association for Computing Machinery (ACM) \\ mateus1996oliveira@hotmail.com, \\ \{joslaine, gecirlei,bispojr\}@ufg.br
}

\begin{abstract}
Algorithm visualization tools (AVT) contribute to learn through stepby-step code visualization. The GraphViewer AVT collaborates in understanding of induction proofs and concepts in Graph Theory. However, a methodological gap has been identified in the validation of its results in the literature. The aim of this work is to highlight and discuss the validation by users of the GraphViewer AVT. Two experiments were carried out. In the first, the test group reached learning gains of almost double the gain obtained by the control group. In the second, the difference was not so significant. It was also found that user acceptance in relation to AVT usability obtained $79.61 \%$ positive evaluations.
\end{abstract}

Resumo. Sistemas de visualização de algoritmos (SVA) auxiliam a aprendizagem por meio da visualização passo-a-passo do código. O SVA GraphViewer colabora na compreensão da demonstração por indução e dos conceitos em Teoria dos Grafos. Porém, foi identificada uma lacuna metodológica na validação dos seus resultados na literatura. O objetivo deste trabalho é evidenciar e discutir a validação pelos usuários do SVA GraphViewer. Dois experimentos foram realizados. No primeiro, o grupo de teste garantiu ganhos de aprendizagem de quase o dobro do ganho obtido pelo grupo de controle. Já no segundo, não foi tão expressiva a diferença. Também foi constatada a aceitação dos usuários em relação à usabilidade do SVA, tendo 79,61\% de avaliações positivas.

\section{Introdução}

Os algoritmos em grafos é tópico de interesse em cursos de graduação em Computação. Porém, uma das dificuldades encontradas por estudantes de Teoria dos Grafos (TG) é a realização de demonstrações de teoremas [Santos e Costa 2007]. Grande parte dos alunos, por exemplo, sente dificuldades em visualizar e utilizar de forma correta a demonstração por indução.

Uma forma de contornar esse problema é atualizando a didática de ensino em um âmbito geral, transformando processos abstratos em concretos [Santos et al. 2008]. Essa atualização pode ser realizada por meio de softwares que possibilitam melhor compreensão dos tópicos abordados e maior interação entre aluno e objeto de estudo, inclusive 
na Educação em Computação [Bispo Jr et al. 2020]. Há ferramentas sendo utilizadas com esse intuito, para visualizar o passo-a-passo de algoritmos prontos [Cordeiro et al. 2004]. Essas ferramentas são chamadas de Sistemas de Visualização de Algoritmos (SVA) [Fincher e Petre 2004].

Em [Carvalho et al. 2017], foi explorado este recorte de pesquisa através da criação do SVA GraphViewer ${ }^{1}$. Foi proposto um método de ensino e aprendizagem com o intuito de auxiliar os alunos que tivessem dificuldades no entendimento e compreensão tanto da técnica de demonstração por indução, quanto dos conceitos abordados na disciplina. Entretanto, uma das fragilidades da proposta foi a validação estatística da metodologia com o uso desse SVA. Conforme mencionado pelos autores, em suas considerações finais, há uma necessidade de melhorar a qualidade do experimento para que, estatisticamente, os resultados tenham maior validade.

Esse trabalho tem como objetivo principal realizar um aperfeiçoamento da validação estatística do experimento realizado em [Carvalho et al. 2017]. O restante do trabalho está dividido da seguinte forma. Na Seção 2, são apresentados os fundamentos deste trabalho. Na Seção 3, os trabalhos relacionados são elencados e comparados. Na Seção 4, a metodologia utilizada no trabalho e os procedimentos são descritos. Na Seção 5, temos a discussão dos resultados obtidos. E, por fim, na Seção 6, as considerações finais são elencadas e possíveis trabalhos futuros.

\section{Fundamentos}

Nesta seção, serão apresentados conceitos fundamentais utilizados neste trabalho. Na Seção 2.1, abordam-se os sistemas de visualização de algoritmos (SVA). Na Seção 2.2, apresentam-se alguns métodos de avaliação de SVA. E, na Seção 2.3, o procedimento de teste de hipótese para dois conjuntos de amostras dependentes.

\subsection{Sistemas de Visualização de Algoritmos}

Os Sistemas de Visualização de Algoritmos (SVA) têm como um de seus objetivos proporcionar ao discente uma experiência mais agradável sobre programação e estudo de algoritmos. Nesses sistemas, é possível visualizar a execução de algoritmos, abstraindoos por meio de uma visualização gráfica da sua execução.

Existem diversos SVA que foram construídos para o ensino de algoritmos e programação. No entanto, cada SVA apresenta um foco relacionado às necessidades específicas de seus usuários. Os SVA utilizam certas linguagens de representação (e.g. fluxograma, grafos) para representar a estrutura do algoritmo. A visualização desempenha um papel de enriquecer a percepção do aluno sobre o alcance dos conceitos estudados.

Independentemente de ser usada uma linguagem convencional ou pseudocódigo, a ferramenta deve permitir a tradução do algoritmo expresso em uma linguagem gráfica para uma forma de representação do código-fonte [Manso et al. 2009]. Existem vários SVA, dos quais podemos mencionar o VisuAlg [Souza 2009], EasyGrafos [Picoli et al. 2019] e o Graph Viewer [Carvalho et al. 2017] (ver Figura 1).

\footnotetext{
${ }^{1}$ Disponível em http://visual-graph.herokuapp.com/.
} 
Figura 1. Captura da tela do SVA GraphViewer durante uma simulação.
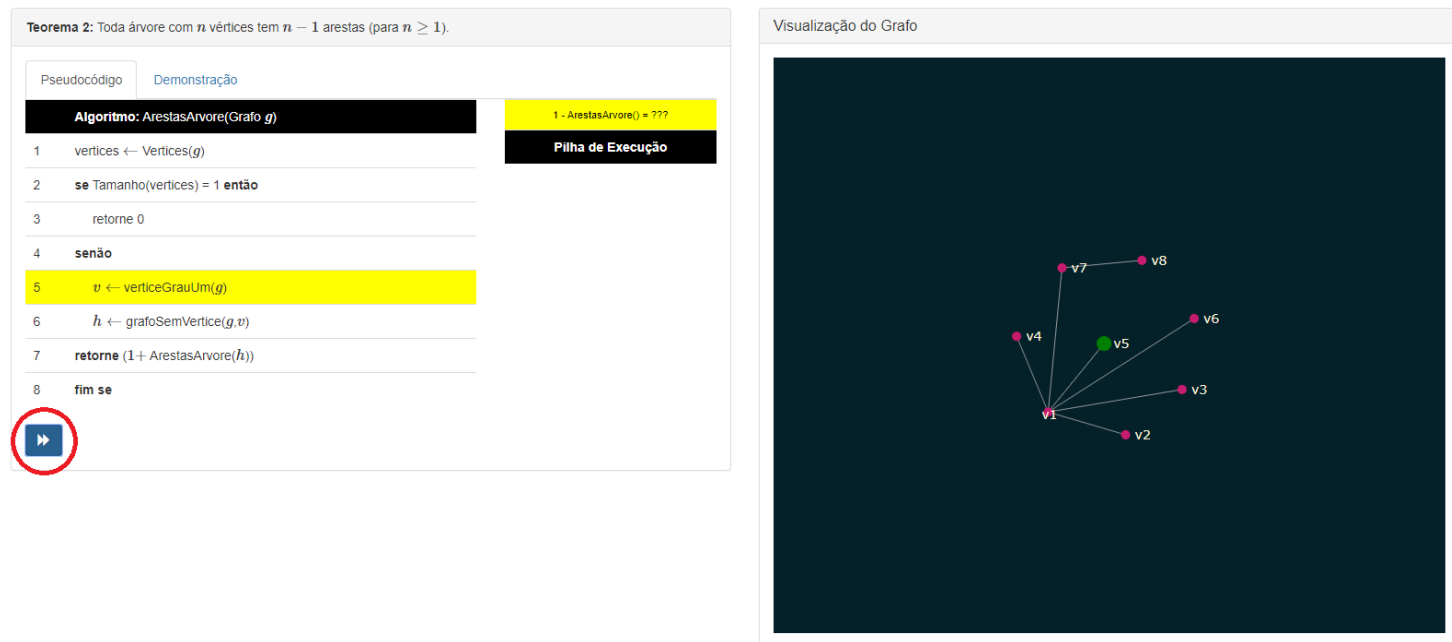

Fonte: Próprio autor.

\subsection{Métricas para Ganho de Aprendizagem}

Métricas de ganhos de aprendizagem são utilizadas para medir os ganhos de forma quantitativa. Elas consideram a quantidade de acertos inicial e a quantidade de acertos após determinada intervenção, no nosso caso o impacto do uso de um SVA. Esse trabalho utiliza duas métricas: a de Ganho Absoluto e de Ganho Normalizado.

Define-se $Q_{\text {pré }}$ e $Q_{\text {pós }}$ como a média obtida por um grupo ao responder o questionário $Q$ realizado anteriormente e posteriormente a um dado momento do experimento, respectivamente. Assim, define-se o ganho de aprendizagem absoluta (GAA) como sendo $G A A=Q_{\text {pós }}-Q_{\text {pré }}$.

A ideia subjacente desta métrica é aferir o ganho de aprendizagem após uma dada intervenção. Suponha que uma determinada turma obteve $Q_{p r e ́}=51 \%$ e, após o uso de um SVA, obteve $Q_{\text {pós }}=72 \%$. Logo, seu GAA seria $21 \%$.

O ganho de aprendizado normalizado (GAN) [Hake 1998, p. 3] fornece uma medida de como o desempenho do estudante cresce, se comparado com o maior crescimento possível que ele possa obter. O GAN é calculado como sendo $G A N=$ $\left(Q_{\text {pós }}-Q_{\text {pré}}\right) /\left(100 \%-Q_{\text {pré}}\right)$.

No exemplo dado na seção anterior, enquanto que o GAA seria $21 \%$, o GAN seria $(72 \%-51 \%) /(100 \%-51 \%) \cong 42,86 \%$. Assim, de todo o ganho de aprendizagem restante "disponível", aproximadamente $42,86 \%$ foi obtido através da intervenção.

\subsection{Testes Estatísticos}

Utilizamos dois questionários para medir quantitativamente os acertos dos alunos em duas situações, antes e após a intervenção. Para cada aluno, temos uma nota adquirida, possibilitando calcular os ganhos obtidos. Existem testes para o nível de significância dos resultados obtidos dessa forma (em duas situações diferentes com o mesmo grupo de pessoas), chamados de testes pareados. Eles utilizam os resultados do GAA para testar se são estatisticamente significativos em relação à população da amostra. 
Os testes paramétricos têm duas condições que devem ser certificadas simultaneamente antes de serem executados, sendo: (1) as variáveis devem possuir uma distribuição normal, e (2) as variâncias populacionais devem ser homogêneas, caso esteja-se comparando duas (ou mais) populações [Marôco 2007].

Sendo assim, quando se tem uma população em que foram extraídos dois conjuntos de amostras emparelhadas, relativas à uma variável dependente quantitativa, utiliza-se o teste $t$-Student pareado. Esse teste é utilizado no projeto para testar a significância dos resultados obtidos em relação ao GAA.

\section{Trabalhos Relacionados}

Para realizar a análise dos trabalhos relacionados, utilizou-se de cinco aspectos: (A1) o foco em ensino e aprendizagem de demonstrações; (A2) o foco em ensino e aprendizagem de TG; (A3) a visualização passo-a-passo dos algoritmos; (A4) a disponibilização do SVA como código-livre; e (A5) a validação estatística mais rigorosa do trabalho. Esse último aspecto, tem as seguinte divisões: (a) validação em relação ao ganho de aprendizagem utilizando o SVA; (b) validação em relação à usabilidade do SVA; e (c) experimento realizado com mais de 25 participantes. A Tabela 1 apresenta a comparação entre os trabalhos relacionados e a proposta deste trabalho.

O A-Graph é um software livre com código fonte aberto que auxilia no ensino e aprendizado da disciplina de TG e seus algoritmos [Lozada 2014]. O EasyGrafos é um protótipo que tem como objetivo ser uma aplicação desktop permitindo ao professor um SVA para auxiliar nos ensinos que inclua TG [Picoli et al. 2019]. Furb Graphs é um SVA de apoio ao ensino de TG com o foco em algoritmos de busca em largura [Bernardes 2016]. Por fim, o GraphViewer é uma ferramenta com âmbito em demonstrações por indução em TG, diferente de todas as outras ferramentas apresentadas. Contendo dois teoremas passíveis de serem demonstrados por indução, para cada um dos teoremas existe um algoritmo recursivo associado e é apresentado visualmente a prova por indução com um pseudocódigo e a visualização do grafo passo-a-passo [Carvalho et al. 2017].

Tabela 1. Tabela comparativa dos trabalhos relacionados, utilizando os cinco aspectos escolhidos (A1 a A5).

\begin{tabular}{l|c|c|c|c|c|c|c|}
\hline & \multicolumn{6}{|c|}{ Aspectos dos SVA } \\
\hline Artigos & A1 & A2 & A3 & A4 & \multicolumn{3}{|c|}{ A5 } \\
\cline { 4 - 8 } & & & a & b & c \\
\hline A-Graph [Lozada 2014] & - & $\sqrt{ }$ & - & $\sqrt{ }$ & - & - & - \\
FURB Graphs [Bernardes 2016] & - & $\sqrt{ }$ & $\sqrt{ }$ & - & - & $\sqrt{ }$ & - \\
EasyGrafos [Picoli et al. 2019] & - & $\sqrt{ }$ & $\sqrt{ }$ & - & - & - & - \\
GraphViewer [Carvalho et al. 2017] & $\sqrt{ }$ & $\sqrt{ }$ & $\sqrt{ }$ & $\sqrt{ }$ & $\sqrt{ }$ & $\sqrt{ }$ & - \\
\hline Proposta deste Trabalho & $\sqrt{ }$ & $\sqrt{ }$ & $\sqrt{ }$ & $\sqrt{ }$ & $\sqrt{ }$ & $\sqrt{ }$ & $\sqrt{ }$ \\
\hline
\end{tabular}

Fonte: Próprio autor. 
IX Congresso Brasileiro de Informática na Educação (CBIE 2020)

Anais do XXXI Simpósio Brasileiro de Informática na Educação (SBIE 2020)

\section{Metodologia}

A metodologia adotada consistiu na utilização do SVA GraphViewer em um ambiente controlado. O experimento foi realizado no Câmpus Jatobá da Regional Jataí, na Universidade Federal de Goiás, em um laboratório de computação.

Os dois experimentos aconteceu em dois períodos, sendo o Experimento 1 e o Experimento 2. O Experimento 1 foi realizado na aula de Probabilidade e Estatística no dia 07/11/2018, contando com a participação de 18 alunos voluntários e um professor voluntário do curso de Computação. O Experimento 2 foi realizado na aula de TG no dia 14/05/2019, contando com a participação de 35 alunos voluntários e um professor voluntário do curso de Computação. Os dois experimentos totalizaram 53 alunos voluntários, alcançando um dos objetivos deste trabalho que era uma amostra de pelo menos 50 participantes. O professor voluntário foi o mesmo em ambos os experimentos.

Ambos experimentos foram divididos da seguinte forma. Separou-se os alunos em dois grupos A e B, sendo respectivamente grupo de controle e grupo de teste. Foram adotados alguns critérios para garantir a distribuição uniforme dos grupos de controle e de teste [Marconi e Lakatos 2003]. Assim, com intuito de garantir a isonomia nos experimentos, os dois grupos foram uniformemente distribuídos seguindo alguns critérios. Os critérios foram aplicados nesta ordem: (i) sexo biológico; (ii) vínculo empregatício; (iii) conhecimento anterior do conteúdo.

Todo o conteúdo ministrado no experimento foi lecionado pelo professor voluntário aos dois grupos simultaneamente. Entretanto, apenas o Grupo B pôde utilizar o GraphViewer durante todo o experimento.

Dois questionários (Questionários 1 e 2) serviram para identificar aspectos sobre a compreensão do estudante a respeito das demonstrações por indução apresentadas para os Teoremas 1 e 2 , respectivamente ${ }^{2}$. Cada um destes questionários teve cinco questões objetivas.

Por fim, o Questionário 3 serviu para identificar aspectos sobre a usabilidade do SVA GraphViewer. Questões sobre facilidade de aprendizado da ferramenta, eficiência de uso, frequência de ocorrência e seriedade dos erros são exemplos dos dados requisitados. Este questionário consiste de cinco perguntas objetivas utilizando uma escala do tipo Likert, com pontuações de um a cinco pontos, respectivamente com rótulos: (i) Discordo Totalmente, (ii) Discordo Parcialmente; (iii) Indiferente, (iv) Concordo parcialmente, e (v) Concordo plenamente.

Cada experimento é dividido em nove momentos:

Momento 1 - aplicação de um questionário socio acadêmico com os participantes;

Momento 2 - aplicação do Questionário 1 (pré-teste) para ambos os grupos;

Momento 3 - exposição, aos dois grupos, de conceitos básicos de grafos e também de demonstração por indução;

Momento 4 - exposição da demonstração por indução do Teorema 1;

\footnotetext{
${ }^{2}$ Todos os questionários e os teoremas desse trabalho podem ser consultados em mais detalhes em https://github.com/bispojr/suplementos-sbie2020-graphviewer
} 
Momento 5 - aplicação do Questionário 1 (pós-teste) para ambos os grupos;

Momento 6 - aplicação do Questionário 2 (pré-teste) para ambos os grupos;

Momento 7 - exposição da demonstração por indução do Teorema 2;

Momento 8 - aplicação do Questionário 2 (pós-teste) para ambos os grupos;

Momento 9 - aplicação do Questionário 3 para o Grupo B.

O paradigma Goal-Question-Metric (GQM) [Van Solingen et al. 2002] foi utilizado no trabalho, através de suas especificações. O GQM é utilizado na Engenharia de Software para auxiliar na medição de processos e softwares, sendo uma abordagem orientada a métricas. A vantagem da sua utilização é que ele separa as preocupações organizacionais (objetivos) das específicas do processo (questões). Isto fornece uma base para decidir quais dados devem ser coletados e como eles devem ser analisados de forma a responder às questões que se pretende resolver. Embora o GQM seja largamente utilizado na Engenharia de Software, esse paradigma vem sendo utilizado e adaptado em pesquisas na área de educação [Gladcheff et al. 2001, Carvalho et al. 2017].

As especificações concretas para os três conceitos principais do GQM foram estabelecidas. O objetivo do projeto consiste em avaliar a utilidade e qualidade do GraphViewer, com ênfase no ensino e aprendizagem de demonstração por indução. Duas questões foram elencadas como refinamentos do objetivo proposto. A primeira é (i) "o GraphViewer melhorou o aprendizado do alunos em relação ao não uso dele?"; e a segunda é (ii) "o GraphViewer é um software fácil de ser utilizado?".

Por fim, métricas são construídas para as duas questões elencadas. Para as especificações das métricas, foram criados quatro questionários. Estes questionários foram submetidos aos participantes do experimento ${ }^{3}$. As respostas concedidas aos questionários serviram de entrada para o cálculo das métricas.

A partir das respostas obtidas dos questionários, duas métricas foram propostas. A métrica M1 é um indicador do impacto na aprendizagem dos estudantes pelo o uso do GraphViewer. A M1 é dada pelos cálculos do GAA e GAN. A seguinte interpretação de M1 foi adotada: se os GAA e GAN forem maiores no grupo de teste em relação ao grupo de controle, então o GraphViewer contribuiu positivamente no aprendizado dos participantes. Caso contrário, a contribuição da ferramenta em relação à aprendizagem pode estar comprometida.

A métrica M2 é um indicador para a usabilidade do GraphViewer. M2 é definida a partir das respostas coletadas através do Questionário 3, que está associado aos aspectos de usabilidade. M2 é obtida pela média da pontuação de todas as respostas de cada participante e varia entre 1,0 e 5,0. A seguinte interpretação de M2 foi adotada: se M2 $>$ 4,0, o que equivale mais de 75,0\% de aceitação do SVA, então GraphViewer é fácil de usar. Caso contrário, o SVA tem a sua usabilidade comprometida.

\section{Resultados}

Na Tabela 2, são apresentados os ganhos de aprendizagem alcançados pelos estudantes durante o Experimento 1. Percebe-se que a utilização do GraphViewer (grupo de teste)

\footnotetext{
${ }^{3} \mathrm{O}$ projeto de pesquisa, o qual esse trabalho está vinculado, foi aprovado pelo Comitê de Ética em Pesquisa sob o $n^{\circ}$ CAAE 79952617.3.0000.5083 e Parecer $n^{\circ} 2540576$.
} 
garantiu ganhos normalizados superiores em todos os cenários. Em relação ao Experimento 1 como um todo, o GAN foi quase o dobro $(25,11 \%)$ do obtido pelo grupo de controle $(13,92 \%)$.

Tabela 2. Ganhos de Aprendizagem Absoluto (GAA) e Normalizado (GAN) em relação aos Teoremas 1 e 2 a partir dos dois grupos de participantes do Experimento 1, do Experimento 2 e dos dois experimentos.

\begin{tabular}{c|c|c|c|c|c|c|c}
\cline { 3 - 7 } & \multicolumn{2}{c}{ Experimento } & \multicolumn{2}{c|}{ Experimento } & \multicolumn{2}{c}{ Dois } \\
& \multicolumn{2}{c}{} & \multicolumn{2}{c}{1} & \multicolumn{2}{c}{2} & \multicolumn{2}{c}{ Experimentos } \\
\cline { 2 - 8 } & Grupo & GAA & GAN & GAA & GAN & GAA & GAN \\
\hline \multirow{2}{*}{ Teorema 1 } & Controle & $2,78 \%$ & $3,70 \%$ & $4,44 \%$ & $6,78 \%$ & $3,70 \%$ & $5,26 \%$ \\
& Teste & $16,67 \%$ & $22,22 \%$ & $9,41 \%$ & $13,79 \%$ & $10,77 \%$ & $14,89 \%$ \\
\hline \multirow{2}{*}{ Teorema 2 } & Controle & $19,44 \%$ & $24,14 \%$ & $13,33 \%$ & $22,22 \%$ & $14,07 \%$ & $20,25 \%$ \\
& Teste & $19,44 \%$ & $28,00 \%$ & $11,76 \%$ & $21,28 \%$ & $13,08 \%$ & $20,99 \%$ \\
\hline Experimento & Controle & $11,11 \%$ & $13,92 \%$ & $8,88 \%$ & $14,50 \%$ & $8,88 \%$ & $12,95 \%$ \\
Inteiro & Teste & $18,06 \%$ & $25,11 \%$ & $10,58 \%$ & $17,53 \%$ & $11,92 \%$ & $17,94 \%$ \\
\hline
\end{tabular}

Fonte: Próprio autor.

Ainda, na Tabela 2, são apresentados os ganhos de aprendizagem durante o Experimento 2. No Teorema 1, o grupo de teste obteve mais ganhos. Porém ocorreu que diferente no Teorema 2, de forma que o grupo de controle obteve uma ligeira vantagem. Em relação ao Experimento 2 inteiro, pode-se perceber que o GraphViewer garantiu GAAs e GANs melhores do que foi obtido pelo grupo de controle, mas não foi semelhante ao Experimento 1 que o GAN chegou a ser quase o dobro.

Continuando ainda na Tabela 2, são apresentados os ganhos de aprendizagem dos dois experimentos. Percebe-se que o uso do GraphViewer garantiu GANs superiores em todos os cenários. Porém, não houve uma discrepância como no Experimento 1 que chegou a ser quase o dobro. Em relação ao GAA, percebe-se que o uso do SVA também foi superior. Apenas no Teorema 2, o grupo de controle $(14,07 \%)$ teve o GAA um pouco maior do que o grupo de teste $(13,08 \%)$.

Na Tabela 3, é apresentado o resultado do teste $t$ dependente com nível de significância 95\% $(\alpha=0,05)$ em relação aos GAAs do Experimento 1. Na única situação em que poderíamos estimar o impacto do uso do GraphViewer é no Teorema 2. Mas quando aplicamos o teste $t$ pareado nos conjuntos, o grupo de teste do Teorema 2 não teve resultado significativo, apenas o grupo de controle. Logo não é possível a comparação.

Ainda, na Tabela 3, é apresentado o resultado do teste $t$ em relação aos GAAs do Experimento 2. De todos os conjuntos de notas coletados, o único que temos uma diferença média significativa é o grupo de teste do Teorema 2, sendo o único grupo que podemos afirmar estatisticamente que os resultados são significativos.

Continuando ainda, na Tabela 3, é apresentado o resultado do teste $t$ em relação aos GAAs dos dois experimentos juntos. Os grupos em que se pode afirmar estatisticamente que os resultados são significativos são os grupos de controle e de teste do Teorema 2, em que se pode comparar o impacto do uso do GraphViewer. Estatisticamente, o uso (ou não uso) do SVA é indiferente em relação ao Teorema 2, neste contexto. Temos $14,07 \%$ de ganho para grupo de controle e $13,08 \%$ para o grupo de teste. 
IX Congresso Brasileiro de Informática na Educação (CBIE 2020)

Anais do XXXI Simpósio Brasileiro de Informática na Educação (SBIE 2020)

Tabela 3. Teste $t$ dependente com nível de significância $95 \%(\alpha=0,05)$ em relação aos GAAs do Experimento 1, do Experimento 2 e dos dois experimentos.

\begin{tabular}{|c|c|c|c|c|}
\hline & Grupo & $\begin{array}{c}\text { Experimento } \\
1\end{array}$ & $\begin{array}{c}\text { Experimento } \\
2\end{array}$ & $\begin{array}{c}\text { Dois } \\
\text { Experimentos }\end{array}$ \\
\hline \multirow{2}{*}{ Teorema 1} & Controle & - & $x$ & -2 \\
\hline & Teste & $\sqrt{ }$ & $x$ & $\times$ \\
\hline \multirow[t]{2}{*}{ Teorema 2} & Controle & $\sqrt{ }$ & $\times$ & $\sqrt{ }$ \\
\hline & Teste & $\times$ & $\sqrt{ }$ & $\sqrt{ }$ \\
\hline \multirow{2}{*}{$\begin{array}{c}\text { Experimento } \\
\text { Inteiro }\end{array}$} & Controle & - & - & - \\
\hline & Teste & $\sqrt{ }$ & - & - \\
\hline
\end{tabular}

(-) Teste não se aplica, ou

$(\sqrt{ })$ Diferença é significativa, ou $(\times)$ não é significativa.

Fonte: Próprio autor.

Vale ressaltar que esse teste é baseado no GAA. Para realizar o teste utilizando ganho de aprendizagem normalizado, é necessária uma fórmula adaptada para o teste $t$ pareado.

No Momento 9, o Questionário 3 foi respondido pelos participantes nos dois experimentos. Um gráfico dos resultados desse questionário é apresentado na Figura 2. É possível verificar que a maioria dos alunos de ambos os grupos avaliaram positivamente a usabilidade do SVA. O cálculo da métrica M2 foi 4,18, sendo 79,61\% de aprovação, atendendo satisfatoriamente a métrica antes estabelecida $(\mathrm{M} 2>4,0)$.

\section{Considerações Finais}

Este trabalho propôs realizar um aperfeiçoamento da validação estatística do experimento realizado em [Carvalho et al. 2017]. Foram estabelecidos critérios para garantir a uniformidade dos grupos de controle e de teste do experimento. Foram realizados dois experimentos, totalizando mais de 50 estudantes.

Em comparação ao trabalho original, além do já posto, utilizou-se métricas para o GAA e GAN e foi feita uma avaliação inicial a respeito das significâncias estatísticas dos resultados obtidos em relação aos GAAs. Infelizmente, em alguns resultados obtidos, não se pode apontar que são significativos por não seguirem uma distribuição normal, e com isso, seria necessário realizar o teste paramétrico $t$, para amostras dependentes.

Em relação aos resultados obtidos, os únicos resultados que passaram em todos os testes e foi possível comparar é o do Teorema 2 com os dois experimentos em conjunto. Porém, foi onde os resultados não tiveram muita diferença. Em relação a essa parte do experimento, utilizar ou não o SVA GraphViewer, é irrelevante estatisticamente falando.

Já nos experimentos isoladamente, mesmo que o uso do GraphViewer apresentasse ganhos que apontam sua relevância, não se pode afirmar que foram ganhos significativos. E em relação à usabilidade, o SVA GraphViewer foi muito bem avaliado em todos os quesitos, tendo poucos votos negativos.

Para extensão deste trabalho, é necessário fazer uma avaliação mais aprofundada 
Figura 2. Gráfico dos resultados do Questionário de Usabilidade do GraphViewer obtidos com os 26 participantes do grupo de teste dos dois experimentos.

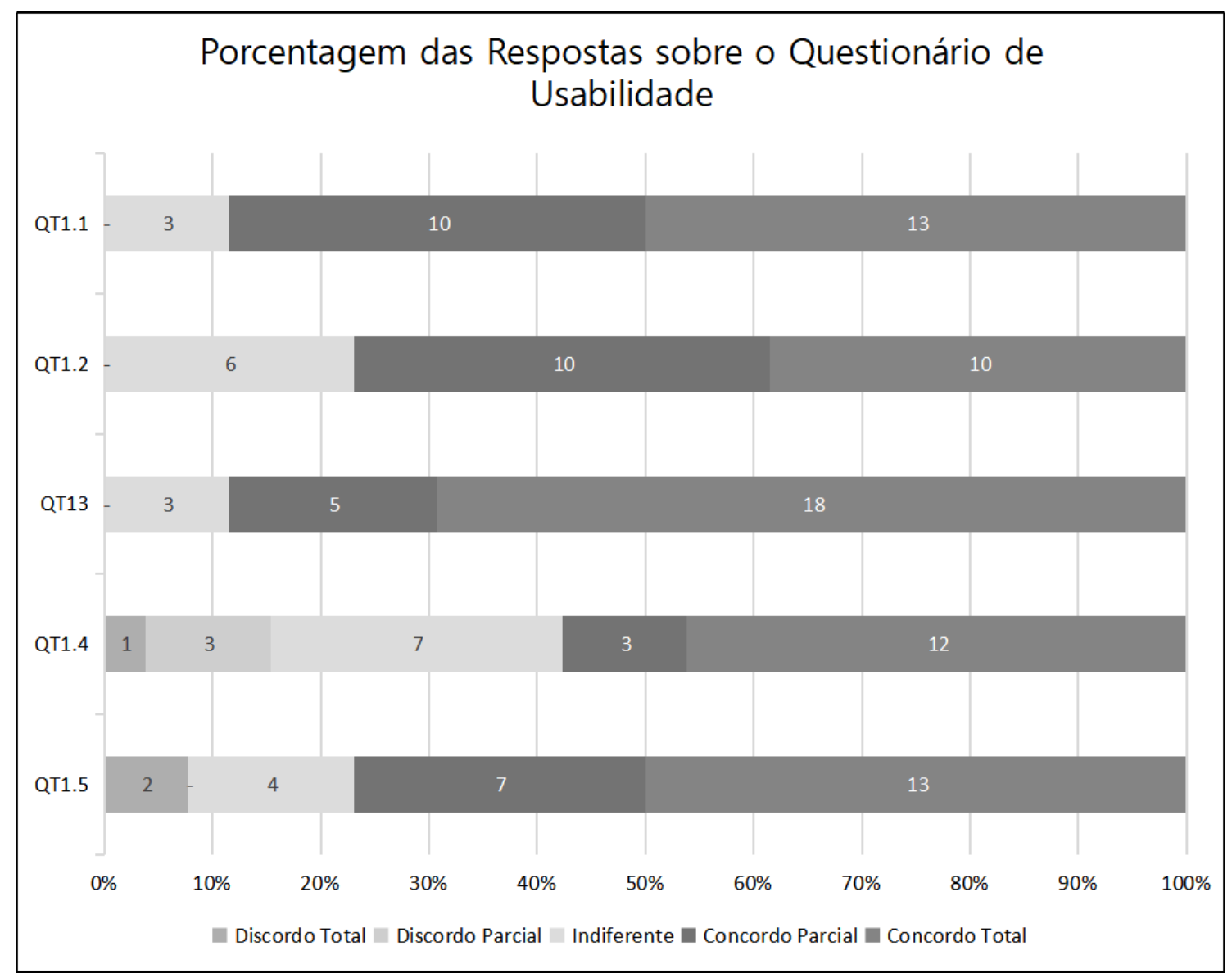

Fonte: Próprio autor.

usando os testes não paramétricos. Assim, é possível descobrir a significância dos ganhos obtidos (inclusive em relação ao GAN). Outra necessidade seria fazer o experimento fora da Universidade Federal de Goiás, com intuito de alcançar outros alunos e outros professores voluntários. Também houve alguns participantes que discordaram das Questões 1.4 e 1.5 (ver Figura 2), sendo duas questões passiveis de serem analisadas em trabalhos futuros para melhor satisfazer o usuário em relação ao SVA.

\section{Referências}

Bernardes, L. H. (2016). FURB Graphs: uma ferramenta de apoio ao aprendizado para a disciplina de teoria dos grafos. Monografia, Universidade Regional de Blumenau.

Bispo Jr, E., Raabe, A., Matos, E., Maschio, E., Barbosa, E., Carvalho, L., Bittencourt, R., Duran, R., e Falcão, T. (2020). Tecnologias na educação em computação: Primeiros referenciais. Revista Brasileira de Informática na Educação, 28:509-527.

Carvalho, F. A., Borges, F. F., Silva, G. F., Borges, T. O., e Bispo Jr, E. L. (2017). Ensino de Provas por Indução em Grafos utilizando uma Ferramenta Visual de Algoritmos. XXII Conferência Internacional sobre Informática na Educação (TISE). 
Cordeiro, E., Stefani, I., Soares, T., e Tirelo, F. (2004). Rin'g: Um ambiente nao-intrusivo para animação de algoritmos em grafos. In Anais do XII Workshop de Educação em Computação (WEI) do Congresso da Sociedade Brasileira de Computação (CSBC) 2004, volume 1.

Fincher, S. e Petre, M. (2004). Algorithm visualization. Computer Science Education Research.

Gladcheff, A. P., Sanches, R., e da Silva, D. M. (2001). Um instrumento de avaliação de qualidade de software educacional: como elaborá-lo. Pensamento \& Realidade. Revista do Programa de Estudos Pós-Graduados em Administração - FEA, 11.

Hake, R. R. (1998). Interactive-engagement versus traditional methods: A six-thousandstudent survey of mechanics test data for introductory physics courses. American Journal of Physics, 66(1):64-74.

Lozada, L. A. P. (2014). A-Graph: Uma ferramenta computacional de suporte para o ensino-aprendizado da disciplina Teoria dos Grafos e seus Algoritmos. In Anais dos Workshops do Congresso Brasileiro de Informática na Educação, volume 3, page 61.

Manso, A., Oliveira, L., e Marques, C. G. (2009). Ambiente de aprendizagem de algoritmos - Portugol IDE. In VI Conferência Internacional de TIC na Educação, pages 969-983.

Marconi, M. d. A. e Lakatos, E. M. (2003). Fundamentos de metodologia científica. Atlas.

Marôco, J. (2007). Análise Estatística com Utilização do SPSS.: $3^{a}$ edição. Súlabo, Lda.

Picoli, J. G., dos Santos, R. V. M., e Mendes, T. M. (2019). Proposta de uma ferramenta computacional para facilitar a aprendizagem sobre caminhamento em grafos. Brazilian Applied Science Review, 3(1):712-720.

Santos, R. P., Costa, H. A., Resende, A. M., e Souza, J. M. (2008). O uso de ambientes gráficos para ensino e aprendizagem de estruturas de dados e de algoritmos em grafos. In Anais do XVI Workshop sobre Educação em Computação, XXVIII Congresso da Sociedade Brasileira de Computação, pages 157-166.

Santos, R. P. e Costa, H. A. X. (2007). TBC-GRAFOS/WEB-treinamento baseado em computador para algoritmos em grafos via web.

Souza, C. M. (2009). VisuAlg - Ferramenta de apoio ao ensino de programação. Revista Eletrônica TECCEN, 2(2):01-09.

Van Solingen, R., Basili, V., Caldiera, G., e Rombach, H. D. (2002). Goal-QuestionMetric (GQM) approach. Encyclopedia of software engineering. 